\section{Cautious reaction to 'super-disasters' prediction}

A dangerous mix of climate change and deepening poverty will create "a decade of super-disasters" and epidemics for the world, according to a new report from the Red Cross. The organization says that so-called natural phenomena, such as earthquakes, droughts, storms and floods triggered by El Niño events, did more damage in 1998 than ever before and displaced 25 million people, more than wars and conflict.

But the World Disasters Report 1999, published by the Geneva-based International Federation of Red Cross and Red Crescent Societies, has drawn a cautious response from experts on its particular claims about disease.

It repeats broad predictions made over the last four years about the effect of global warming on vector-borne diseases. For example, it quotes models developed by the Intergovernmental Panel on Cli- mate Change, suggesting that by 2100 there could be between 50 and 80 million extra cases of malaria and 3.5 million cases of river blindness due to climate change.

David Heymann, head of the communicable diseases program at the World Health Organization (WHO), accepts that climate change is likely to have adverse effects on health, but says that the WHO has not made specific projections about the size of those effects. "We believe it is early days to make predictions," he says.

Certain diseases, including malaria, rift valley fever, meningitis, cholera and other diarrheal infections, are sensitive to climate change, but the spread of other important human killers such as tuberculosis is comparatively unaffected by climate and more closely linked to factors such as increasing antimicrobial resistance, says Heymann.

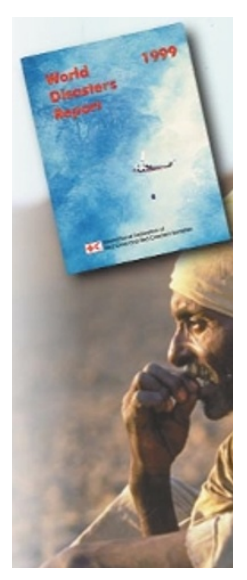

Malaria expert Brian Greenwood of the London School of Hygiene and Tropical Medicine, agrees that increased transmission of the disease has been linked to global warming, for example in upland Tanzania, or after floods triggered by El Niño. But compared with the impact of wars, civil disorder and health-system breakdown in countries such as the Democratic Republic of Congo, the numbers affected are small. " I think that more Africans are suffering malaria because of breakdown in society than because of climate change," he says. A copy of the report is available at www.ifrc.org

PHYLlida BRown, LONDON

\section{California aims to direct global health}

The Universitites of California at San Francisco (UCSF) and Berkeley (UCB) have joined forces to establish the Institute for Global Health (IGH) to tackle international health policy, vaccine development and distribution and reproductive health. IGH, which will open for business next month in downtown San Francisco, will also feature an interdisciplinary basic research program and a training program for policy makers.

"The concentration of universities and relevant companies in the Bay Area makes

\section{Japan tries to improve clinical trial numbers}

The Japanese Ministry of Health and Welfare (MHW) has announced a set of measures to counteract a drastic downturn in the number of clinical trials in the country.

Following a report by an ad-hoc expert panel released last month, MHW is to support the training of dedicated personnel for the coordination and oversight of clinical trials, and assist hospitals in upgrading their clinical research infrastructure. To facilitate patient enrollment, pharmaceutical companies will be allowed to advertise trials and provide financial incentives to participants.

The total number of clinical trial protocols filed to MHW has decreased to under a third over the past five years following the enforcement of new good clinical practice (GCP) standards mandating strict informed consent rules. Fumimaro Takaku, president of the Jiji Medical School and a prominent MHW advisor describes the hollowing out of clinical trials in Japan as a "very serious issue," and points out that before the new GCP standard was enacted, "patients and their families were rarely informed of their participation in a clinical trial," which may account for the present downturn.

Takaku explains that the administrative load on hospitals conducting clinical trials has increased considerably with the new GCP rules and hospitals were ill prepared to cope with the new rules. Consequently, many Japanese pharmaceutical companies have transferred an increasing part of their clinical testing in Europe or the United States.

Robert TriendL, Tokyo
World Bank (WB) in Washington DC, where he ran an annual $\$ 15$ billion lending program to 85 countries for four years. Before that, he was the dean of the London School of Hygiene and Tropical Medicine for six years. "The sole reason we will work with and trust IGH is Richard's reputation," states Ed Penhoet, the dean of UCB's School of Public Health and the vice-chairman of Chiron, a leading biotechnology firm in nearby Emeryville.

And Helen Saxenian, a WB sector manager for health nutrition population and a former colleague of Feachem's, adds that, although the WB has "no current plans to work with IGH, we certainly would draw on its expertise because of Richard."

IGH will develop new vaccines-initially for pneumonia, HIV, tuberculosis, malaria, and hepatitis $\mathrm{C}$-and then plans to work with major international agencies, including WB and the World Health Organization, to distribute them.

"Right now, vaccines are the best buy in the huge supermarket of health products," explains Feachem. "They buy you the most years of healthy life per dollar spent. But many of the bureaucrats who control public-health funds in the developing countries don't realize this. IGH will do its level best to educate these officials."

\section{B.J. Spalding, San Francisco}

